\title{
Immunization of adult dairy cattle with a new heat-killed vaccine is associated with longer productive life prior to cows being sent to slaughter with suspected paratuberculosis
}

\author{
M. Alonso-Hearn, E. Molina, M. Geijo, P. Vazquez, I. A. Sevilla, J. M. Garrido, and R. A. Juste ${ }^{1}$ \\ Department of Animal Health, NEIKER-Tecnalia, Derio, Bizkaia 48160, Spain
}

\begin{abstract}
The objective of this study was to evaluate the efficacy of a new heat-killed Mycobacterium avium ssp. paratuberculosis (MAP) vaccine for control of premature culling in tuberculosis-free dairy cattle. Feces and gastrointestinal tissues were collected from 50 vaccinated cows and 38 nonvaccinated cows at slaughter and analyzed by bacteriological culture and histopathology. Vaccination was associated with a significant reduction of the frequency of vaccinated animals with MAP in feces and gut tissues compared with the nonvaccinated animals. In addition, the frequency of vaccinated animals with heavy bacterial load in gut tissues was $40 \%$ lower than the frequency of the nonvaccinated animals with the same MAP load. The peak age of paratuberculosis-associated culling was from 4.5 to 5 yr old $(21 \%)$ in the vaccinated animals and from 3 to $4.5 \mathrm{yr}$ old $(60 \%)$ in the nonvaccinated animals. The vaccinated and nonvaccinated animals with suspected paratuberculosis were culled at an average age of 4.7 and $3.7 \mathrm{yr}$ old, respectively. Therefore, a significant increase in the productive life of the vaccinated animals sent to slaughter with suspected paratuberculosis was observed. In addition, our analysis revealed a positive effect of the vaccine on the carcass weights of the animals with severe histopathological lesions at slaughter compared with the nonvaccinated animals. In summary, our findings suggest a therapeutic effect of the vaccine and a significant attenuation of pre-existing infection in cows naturally infected with paratuberculosis that were adults at the time of vaccination.
\end{abstract}

Key words: dairy cow, paratuberculosis, infection, vaccination, culling

\section{INTRODUCTION}

Paratuberculosis or Johne's disease is an inflammatory bowel disease of ruminants caused by Mycobacte-

Received October 28, 2009.

Accepted October 5, 2011.

${ }^{1}$ Corresponding author: rjuste@neiker.net rium avium ssp. paratuberculosis (MAP) and that is responsible for considerable economic losses to the dairy cattle industry because of reduction in milk production, premature culling, lower slaughter value, and increased cow replacement rates (Ott et al., 1999). Mycobacterium avium ssp. paratuberculosis is of interest because of its possible involvement in human Crohn's disease, a chronic debilitating disease of unknown etiology that may resemble, in some aspects, Johne's disease. Although several studies have reported the presence of MAP in blood, breast milk, and intestinal tissues of some Crohn's disease patients, it is still not widely accepted that MAP causes Crohn's disease (Chamberlin et al., 2001; Juste et al., 2008, 2009b; Rosenfeld and Bressler, 2010).

The most important mode of transmission of paratuberculosis is ingestion of MAP-contaminated milk, colostrum, feed materials, or water (Daniels et al., 2003; Nielsen et al., 2008; Pierce, 2009). Because calves rarely shed detectable levels of MAP in feces and therefore are less infectious than adult cattle, frequent contact between calves and cows is an important factor in the spread of MAP in infected herds (Sweeney et al., 1996). Paratuberculosis is a slowly progressive chronic infection, with most clinical cases occurring between 3 and 5 yr of age (Chiodini et al., 1984). For most animals, the disease is restricted to a subclinical infection; however, animals that progress to a clinical stage gradually lose weight and are culled from the herd (Collins, 2003). Herds with 1 to $5 \%$ of clinically affected cows could have up to $50 \%$ of subclinical carriers and asymptomatic shedders (Sherman et al., 1990).

Eradication programs based on the testing and culling of infected animals have been unsuccessful due to the lack of diagnostic tests able to detect MAP in the early stages of the infection (Manning and Collins, 2001). Control programs based on vaccination with heat-killed vaccines against paratuberculosis were implemented in France and the Netherlands and are still in use in Australia, the United Kingdom, and the United States (Argenté, 1992; van Schaik et al., 1996). In Spain, vaccination with killed vaccines against paratuberculosis in sheep and goats has been recommended 
by the animal health authorities to reduce losses in production due to the clinical disease (Juste et al., 1994). However, concerns over the interference of these vaccines with tests for detection of Mycobacterium bovis have restricted their use in cattle (Kohler et al., 2001). The near eradication of bovine tuberculosis in the Basque Country of northern Spain, as well as the high prevalence of clinical cases of paratuberculosis, led the regional Animal Health Department to test the efficacy of a new heat-killed MAP vaccine (Silirum, CZV, Porriño, Spain) in dairy cows naturally infected with MAP. In the present study, we analyzed the effects of the immunization with the Silirum vaccine on (1) the development of clinical signs and lesions associated with a paratuberculosis infection, (2) the amount of bacteria detected in feces and tissues, (3) the age at culling, and (4) the carcass weights of vaccinated and nonvaccinated cows at slaughter. Although a reduction in the number of cattle with clinical evidence of paratuberculosis has been reported after immunization with similar vaccines in other countries (Körmendy, 1994; Wentink et al., 1994), the effects of the vaccination strategy on the control of premature culling in naturally infected dairy cattle had not been analyzed before. To the author's knowledge, this is one of the first studies to report the effects of vaccination of adult cattle.

\section{MATERIALS AND METHODS}

\section{Herds}

A vaccination program for paratuberculosis is being conducted in 5 Holstein-Friesian dairy farms (farms A, B, C, D, E) and 1 Jersey dairy farm (farm F) of the Basque Country. Mean herd size was 87, ranging from 41 to 120 adult cows per herd. At the beginning of the trial, the incidence of cows with farmer-suspected paratuberculosis in the selected herds ranged from 2 to 10\%. Two nonvaccinated Holstein-Friesian herds, with approximately 250 cows in each herd and a similar incidence of farmer-suspected paratuberculosis compared with the vaccinated herds, were used as controls (farms $\mathrm{G}$ and $\mathrm{H}$ ). A description of the 8 herds included in the study, including their breeds, herd sizes, incidence of farmer-suspected paratuberculosis, and the dates at the beginning of the trial are shown in Table 1. The herds were officially free of bovine tuberculosis for at least $5 \mathrm{yr}$ before the start of the vaccination program as determined by the intradermal tuberculin test included in the Spanish control program of bovine tuberculosis. Management practices directed against paratuberculosis such as minimizing fecal contamination of water, feed, milk, and tools; early separation of newborn calves from adult cattle, utilization of colostrum from cows with a negative fecal culture and ELISA tests within the past year and avoiding purchase of unknown health status animals were recommended to all the farmers. Common management practices designed to reduce MAP transmission within each herd were monitored and farmers interviewed regularly to ensure that all the producers implement them in a similar way.

In the vaccinated herds, $1 \mathrm{~mL}$ of the Silirum MAP vaccine $(\mathrm{CZV})$ was administered subcutaneously using a $0.9-\mathrm{mm}$ needle into the dewlap of all calves $(>1$ mo old) and cows at the time of joining the trial, and then to all the new 1- to 2-mo-old replacer calves. Each dose contained $2.5 \mathrm{mg}$ of heat-killed 316F MAP strain plus mineral oil as adjuvant and thiomersal (known as thimerosal in the United States) as a preservative. The vaccination trial was authorized by the Agencia Española de Medicamentos y Productos Sanitarios (AEM No. 107/ECV) and endorsed by the Ministerio de Medio Ambiente y Medio Rural y Marino.

\section{Study Population and Reasons for Culling}

Due to constraints regarding dates and places of slaughter, our study population included only 50 vaccinated and 38 nonvaccinated animals from the 8 herds affected by Johne's disease that could be followed to slaughter. We assumed that the selection method was representative and similar for both treatment groups. This sample represented 15 and $16 \%$ of the total culling of the vaccinated and nonvaccinated farms from the date of initiation of the trial to September 2008, respectively. The nonvaccinated cows included in the study were slaughtered between June 2006 and March 2009 and the vaccinated cows between November 2005 and September 2008.

The 50 vaccinated animals included in the study were sent to slaughter because of farmer-suspected paratuberculosis $(\mathrm{n}=14)$ or low milk production ( $\mathrm{n}$ $=36$ ), in some cases as a result of mastitis, lameness, infertility, septicemia, or old age. The nonvaccinated animals $(\mathrm{n}=38)$ were sent to slaughter for the same reasons: farmer-suspected paratuberculosis $(\mathrm{n}=15)$ or low milk production $(\mathrm{n}=23)$. Vaccinated and nonvaccinated cows were culled due to farmer-suspected paratuberculosis if they showed observable signs associated with this disease, such as persistent diarrhea or weight loss. Animals sent to slaughter only because of low milk production may have had subclinical disease and been asymptomatic carriers. Subclinically infected cows are usually less productive than healthy cows but do not yet exhibit the characteristic signs of Johne's disease such as persistent diarrhea or weight loss. Because our study population was intended to include a substantial number of animals in various stages of 
Table 1. Breed, herd size, incidence of cows with farmer-suspected paratuberculosis at the beginning of the trial, and start dates of the field trial for each herd, number of animals sent to slaughter because of farmer-suspected paratuberculosis or low milk production, and months from the date of joining the field trial (intervention) to the slaughter of the selected cows for each herd ${ }^{1}$

\begin{tabular}{|c|c|c|c|c|c|c|c|c|}
\hline \multirow[b]{2}{*}{ Farm ID } & \multirow[b]{2}{*}{ Breed } & \multirow[b]{2}{*}{$\begin{array}{l}\text { Herd size } \\
\quad(\text { no. })\end{array}$} & \multirow[b]{2}{*}{$\begin{array}{l}\text { Incidence } \\
\quad(\%)\end{array}$} & \multirow[b]{2}{*}{$\begin{array}{l}\text { Beginning } \\
\text { of the trial }\end{array}$} & \multicolumn{2}{|c|}{$\begin{array}{l}\text { Slaughtered } \\
\text { animals }^{2} \text { (no.) }\end{array}$} & \multicolumn{2}{|c|}{$\begin{array}{l}\text { Mean time (mo } \pm \mathrm{SD}) \text { between } \\
\text { intervention and slaughter (range) }\end{array}$} \\
\hline & & & & & PTBC & No PTBC & Herd & Strategy ${ }^{3}$ \\
\hline \multicolumn{9}{|c|}{ Nonvaccinated } \\
\hline G & Holstein & 250 & 5 & May 30, 2006 & 10 & 3 & $\begin{array}{l}10.5 \pm 10.1 \\
(0.7-25.4)\end{array}$ & \multirow[t]{2}{*}{$\begin{array}{l}14.5 \pm 8.2 \\
(0.7-31.3)\end{array}$} \\
\hline $\mathrm{H}$ & Holstein & 250 & 5 & May 29, 2006 & 5 & 20 & $\begin{array}{l}16.6 \pm 6.3 \\
(4.9-31.3)\end{array}$ & \\
\hline B & Holstein & 100 & 8 & January 17, 2003 & 2 & 0 & \multirow{3}{*}{$\begin{array}{c}59.1 \pm 4.4 \\
(56-62.2) \\
16.8 \pm 8.2 \\
(4.0-30.8) \\
11.8 \pm 9.2 \\
(1.6-30.5) \\
12.0 \pm 6.2 \\
(3.8-24.2) \\
10.2 \pm 0\end{array}$} & \multirow{3}{*}{$\begin{array}{l}15.2 \pm 11.9 \\
(1.6-62.2)\end{array}$} \\
\hline $\mathrm{C}$ & Holstein & 41 & 6 & March 16, 2006 & 5 & 11 & & \\
\hline $\mathrm{D}$ & Holstein & 99 & 3 & March 17, 2006 & 1 & 13 & & \\
\hline
\end{tabular}

${ }^{1}$ For the nonvaccinated herds, the intervention time was the start date of the field trial and for the vaccinated cows it was the vaccination date. ${ }^{2} \mathrm{PTBC}=$ cows sent to slaughter with paratuberculosis-suspected clinical signs (severe diarrhea or weight loss); no PTBC $=$ cows sent to slaughter because of low milk production.

${ }^{3}$ Mean time between intervention and slaughter of all the vaccinated and all of the nonvaccinated cows combined.

infection and a variety of physical conditions, any positive effect of vaccination was evaluated in cows sent to slaughter because of farmer-suspected paratuberculosis or low milk production. Information about age, breed, carcass weight, and reason for culling for each cow was obtained from the Animal Health Services of the Diputacion Foral de Bizkaia and Gipuzkoa (European Union Cattle Database).

For analysis of the age of culling, all vaccinated (n $=332)$ and nonvaccinated animals $(\mathrm{n}=233)$ since the beginning of the trial were used. Nonvaccinated cows were further subdivided into 2 categories: animals with a positive premortem test and animals with a negative premortem test (fecal culture or ELISA tests) within the past year. Antibody titers against MAP were determined by a commercial ELISA according to the instructions provided by the manufacturer (Institut Pourquier, Montpellier, France). The specificity of the ELISA test was $\geq 99.8 \%$ and its sensitivity in detecting fecal culturepositive cattle was 27.8 to $28.9 \%$ (Collins et al., 2005).

\section{Tissue and Fecal Sampling for Histopathology and Bacteriological Culture}

On their corresponding day of slaughter, the 50 vaccinated and the 38 nonvaccinated animals included in this study were examined by slaughterhouses veterinarians and killed by captive-bolt stunning. After exsanguination, the gastrointestinal tracts were tied off, removed from the carcasses, and immediately transported to the laboratory in disposable plastic bags. Before individual tissue collection, tables and instruments were cleaned with soap and $5.25 \%$ sodium hypochlorite. Gloves and sterile scalpels were changed between the handling of each gastrointestinal tract. For histopathology, samples from ileocecal lymph node, jejunal lymph node, ileocecal valve (ICV), jejunum, and terminal ileum were collected aseptically from each animal and placed in formalin within $24 \mathrm{~h}$ after arrival at the laboratory. The samples were preferentially taken from the areas of the preselected tissues that showed paratuberculosis-associated lesions, including thickened mucosa and enlarged lymphatic nodes, if present. The collected samples were fixed in $10 \%$ neutral buffered formalin, embedded in paraffin, and sectioned at $4 \mu \mathrm{m}$. Sections were stained with hematoxylin and eosin and with Ziehl-Neelsen and examined by light microscopy. Paratuberculosis lesions were graded as focal, multifocal, diffuse paucibacillary, diffuse intermediate, or diffuse multibacillary (González et al., 2005).

For bacteriological culture, 4 tissue samples from each animal including a pool of samples from ICV and 3 sites of ileum (proximal, middle, and terminal ileum), a pool of samples from 3 sites of the jejunum (proximal, intermediate, and distal), and 2 separate samples from ileocecal lymph node and jejunal lymph node, were collected. Two grams of each sample were decontaminated with $38 \mathrm{~mL}$ of hexa-decyl pyridinium chloride at a final concentration of $0.75 \%$ (Sigma, St. Louis, MO) and homogenized in a stomacher. After 30 min of incuba- 
tion at room temperature, $15 \mathrm{~mL}$ of the suspension was transferred to a new tube and incubated overnight for decontamination and sedimentation. Four drops were taken from the layer of the suspension near the sediment and inoculated into 2 tubes of Herrold's egg yolk medium (HEYM; Becton Dickinson, Sparks, MD) and 2 tubes of Loewenstein-Jensen medium (L-J; Difco, Detroit, MI), both supplemented with Mycobactin J (Allied Monitor, Fayette, MO; Juste et al., 1991). Tubes were incubated at $37^{\circ} \mathrm{C}$ in a slanted position with loose caps to allow the surface of the medium to dry. After $1 \mathrm{wk}$, caps on the slants were tightened, and cultures were observed every $4 \mathrm{wk}$ and considered negative if no bacterial growth was observed after 20 wk. Samples were considered positive if one or more colony-forming units with morphology typical for MAP were observed in one or more culture tubes. Bacterial load in tissues was classified as low ( $<10 \mathrm{cfu}$; estimated average 2 cfu/tube), medium (10 to $50 \mathrm{cfu}$; estimated average 20 cfu/tube), or heavy (>50 cfu; estimated average 200 cfu/tube). Positive colonies were confirmed by PCR amplification of the IS900 MAP insertion sequence, as described previously (Moss et al., 1992).

At the time of slaughter, feces were taken from the rectum of each animal and processed within $48 \mathrm{~h}$ after arrival at the laboratory. Briefly, pools of fecal samples from 2 cows (2 $\mathrm{g}$ each) were decontaminated, blended in a stomacher, and cultured in HEYM and L-J, as described previously for tissue culture. For pools with a positive culture result, individual fecal samples were processed and cultured. Animals were classified as low $(<10 \mathrm{cfu})$, medium $(10$ to $50 \mathrm{cfu})$, or heavy ( $>50 \mathrm{cfu})$ shedders based on MAP colony-forming units from feces.

\section{Statistical Analysis}

Categorical variables such as the presence of lesions or the detection of MAP in feces and tissues of vaccinated and nonvaccinated animals were compared using a one-tailed Dunnett test after transformation into a numerical binomial variable with the GLM procedure and using a Fisher exact probability test with the FREQ procedure of the SAS software (SAS Institute Inc., Cary, NC). Means of age at culling were compared using a Student's t-test (GraphPad Software, San Diego, CA). The effects of vaccination and the presence of histopathological lesions consistent with paratuberculosis on the age and weight at slaughter were tested by ANOVA with the GLM procedure (SAS Institute Inc.). In the analysis of the effects of the immunopathological forms on carcass weights, age was used as a covariable. $P$-values are shown only when significant differences between groups were observed. When differences between groups were not statistically significant, $P$-values and percentages of reduction or numerical differences between groups are omitted, and only the frequencies of both vaccinated and nonvaccinated cows are shown.

\section{RESULTS}

\section{Vaccinated and Nonvaccinated Cows Sent to Slaughter Because of Farmer-Suspected Paratuberculosis or Low Milk Production}

Fifty vaccinated and 38 nonvaccinated animals from 8 dairy herds affected by Johne's disease were sent to slaughter because of farmer-suspected paratuberculosis or low milk production. The breeds, herd sizes, and frequencies of farmer-suspected paratuberculosis at the beginning of the trial and start dates of the trial for each herd are shown in Table 1. In addition, the table shows the number of animals sent to slaughter because of suspected paratuberculosis or low milk production, and the mean time between the dates of joining the field trial to the slaughter of the selected animals for each herd. This interval was approximately $15 \mathrm{mo}$ in both vaccinated and nonvaccinated herds. Most of the 50 vaccinated cows were adults at the time of vaccination, with only 4 cows vaccinated at less than 24 mo of age and killed at less than $3 \mathrm{yr}$ of age. Because most of the cows included in our study population were vaccinated as adults, the analysis of the data are shown with or without the 4 cows vaccinated as calves.

\section{Histopathological Lesions and Bacteriological Culture of Feces and Gut Tissues of the Cows Sent to Slaughter Because of Farmer-Suspected Paratuberculosis}

Signs of paratuberculosis, including persistent diarrhea and weight loss, were observed in 14 of the 50 vaccinated animals (28\%) and in 15 of the 38 nonvaccinated cows $(39 \%)$. All of the vaccinated animals that were slaughtered with farmer-suspected paratuberculosis were vaccinated at less than 21 mo of age and sent to slaughter at least 11 mo later. Fecal and tissue culture data and the histopathological examination of gut tissues from the 29 cows sent to slaughter with suspected paratuberculosis are shown in Table 2. Signs of paratuberculosis, especially diarrhea, are common among cows with other diseases such as Salmonellosis, renal amyloidosis, parasitism, and ruminal acidosis (Smith, 1990). Nevertheless, in our study, nearly all cows sent to slaughter with farmer-suspected paratuberculosis had a positive paratuberculosis test postmortem as well. As seen in Table $2,86 \%$ of the vaccinated animals $(12 / 14)$ and $100 \%$ of the nonvaccinated animals $(15 / 15)$ had lesions consistent with paratuberculosis in the gut and most of them showed severe, diffuse lesions. In fact, 
Table 2. Bacteriological culture from feces and gastrointestinal tissues, and histopathological findings of the vaccinated and nonvaccinated animals sent to slaughter with farmer-suspected paratuberculosis

\begin{tabular}{|c|c|c|c|c|c|}
\hline Animal code & $\begin{array}{l}\text { Fecal } \\
\text { culture }^{1}\end{array}$ & $\begin{array}{l}\text { Tissue } \\
\text { culture }^{2}\end{array}$ & Histopathology & $\begin{array}{c}\text { Age at } \\
\text { vaccination }(\mathrm{yr})\end{array}$ & $\begin{array}{c}\text { Age at } \\
\text { slaughter (yr) }\end{array}$ \\
\hline Vaccinate-1 & Negative & Negative & No paratuberculosis & 1.77 & 2.73 \\
\hline Vaccinate-3 & Medium & Heavy & Diffuse paucibacillary & 2.37 & 3.35 \\
\hline Vaccinate-4 & Low & Heavy & Diffuse intermediate & 3.30 & 3.88 \\
\hline Vaccinate- 5 & Negative & Heavy & Diffuse intermediate & 2.05 & 4.13 \\
\hline Vaccinate- 6 & $\mathrm{ND}^{3}$ & Heavy & Diffuse multibacillary & 4.18 & 4.51 \\
\hline Vaccinate-9 & Low & Heavy & Multifocal & 3.42 & 5.06 \\
\hline Vaccinate-10 & Low & Heavy & Diffuse intermediate & 3.16 & 5.16 \\
\hline Vaccinate-11 & Heavy & Heavy & Diffuse multibacillary & 0.36 & 5.55 \\
\hline Vaccinate-12 & Heavy & Heavy & Diffuse multibacillary & 4.87 & 5.85 \\
\hline Vaccinate-13 & Negative & Medium & Diffuse multibacillary & 5.63 & 6.17 \\
\hline Vaccinate-14 & Heavy & Heavy & Diffuse intermediate & 4.82 & 6.90 \\
\hline Nonvaccinate-1 & Medium & Heavy & Diffuse intermediate & - & 1.84 \\
\hline Nonvaccinate-7 & Negative & Heavy & Multifocal & - & 3.69 \\
\hline Nonvaccinate- 8 & Heavy & Heavy & Diffuse multibacillary & - & 3.82 \\
\hline Nonvaccinate-9 & Low & Heavy & Diffuse intermediate & - & 3.82 \\
\hline Nonvaccinate-10 & Medium & Heavy & Diffuse paucibacillary & - & 4.08 \\
\hline Nonvaccinate-11 & Medium & Heavy & Diffuse multibacillary & - & 4.16 \\
\hline Nonvaccinate- 12 & Low & Heavy & Multifocal & - & 4.17 \\
\hline Nonvaccinate-13 & Medium & Heavy & Multifocal & - & 4.89 \\
\hline Nonvaccinate-14 & Negative & Negative & Focal & - & 4.92 \\
\hline Nonvaccinate-15 & Low & Heavy & Diffuse multibacillary & - & 6.39 \\
\hline
\end{tabular}

${ }^{1}$ Fecal culture classes were low $(<10 \mathrm{cfu})$, medium $(10$ to $50 \mathrm{cfu})$, and heavy $(>50 \mathrm{cfu})$.

${ }^{2}$ Tissue culture classes were low $(<10 \mathrm{cfu})$, medium (10 to $\left.50 \mathrm{cfu}\right)$, and heavy $(>50 \mathrm{cfu})$.

${ }^{3} \mathrm{ND}=$ not determined

the number of vaccinated and nonvaccinated animals with diffuse lesions was the same in both groups. However, more nonvaccinated cows manifested clinical signs of the disease and had focal or multifocal lesions in gut tissues (4/15) compared with vaccinated cows $(1 / 14)$. Most of the vaccinated $(79 \%)$ and almost all the nonvaccinated cows (93\%) with clinical signs had a heavy MAP load in tissue and shed Map in the feces in sufficient quantity to be detectable. In fact, $64 \%$ of the vaccinated and $80 \%$ of the nonvaccinated cows were fecal-culture positive. Only one cow with farmersuspected paratuberculosis, vaccinate-1, had negative histopathological and culture results and it did not show diarrhea, only gradual weight loss. Average age of slaughter of the vaccinated and nonvaccinated cows with farmer-suspected paratuberculosis was 4.7 and 3.7 yr old, respectively.

\section{Histopathological Lesions of the Cows Sent to Slaughter Because of Farmer-Suspected Paratuberculosis or Low Milk Production}

Although gut tissues were collected from the study population, tissue samples from 5 of the 50 vaccinated cows and from 3 of the 38 nonvaccinated cows showed signs of autolysis, making the histopathological analysis impossible. The results of the histopathological examination of gut tissues from 45 vaccinated cows and from 35 nonvaccinated cows are presented in Table 3. Although not statistically significant, differences were observed in the number of vaccinated cows with lesions compared with the nonvaccinated cows. Fortynine percent of the vaccinated animals and $63 \%$ of the nonvaccinated animals developed paratuberculosisassociated lesions in gut tissues. Thirty-one percent of the vaccinated cows had diffuse lesions versus $43 \%$ of nonvaccinated cows. Diffuse multibacillary forms were observed in $13 \%$ of the vaccinated cows and in $17 \%$ of the nonvaccinated cows.

\section{MAP Detection in Feces and Tissues of the Cows Sent to Slaughter Because of Farmer-Suspected Paratuberculosis or Low Milk Production}

The frequency of slaughtered animals with low, medium, and heavy bacterial load in their feces and tissues is shown in Figure 1. Results of the bacteriological culture from feces revealed that $44 \%$ of the 
nonvaccinated animals were shedding compared with $24 \%$ of the vaccinated cows. As shown in Figure 1A, a $45 \%$ reduction in the frequency of vaccinated animals excreting MAP in their feces was observed compared with the nonvaccinated group (Fisher's test, $P=0.051$; Dunnett test, $P=0.030$ ). If we exclude the 4 cows vaccinated at less than 24 mo of age and killed at less than $3 \mathrm{yr}$ of age, the frequencies of nonvaccinated and vaccinated animals with MAP load in feces were 44 and $27 \%$, respectively.

Results of the bacteriological culture from gut tissues revealed that $71 \%$ of the nonvaccinated cows were infected compared with $52 \%$ of the vaccinated cows. As shown in Figure 1B, vaccination resulted in a $27 \%$ reduction in the number of vaccinated animals with MAP infection in gut tissues (Fisher's test, $P=0.055$; Dunnett test, $P=0.035$; Figure $1 \mathrm{~B}$ ). In addition, the frequency of vaccinated animals with heavy bacterial load (>50 cfu) in gut tissues (30\%) was $40 \%$ lower than the frequency of the nonvaccinated animals with the same MAP load (50\%; Fisher's test, $P=0.045$; Dunnett test, $P=0.028)$. When the cows vaccinated at less than 24 mo of age and killed at less than $3 \mathrm{yr}$ of age were excluded from the analysis, the frequencies of nonvaccinated and vaccinated animals with MAP load in gut tissues were 71 and $54 \%$, respectively. Differences in MAP recovery between HEYM and L-J were observed. All the positive samples from feces or tissues grew in HEYM but none of them was positive only in L-J. Seventy-eight percent of the positive samples from tissues grew in HEYM and LJ, whereas $30.7 \%$ of the fecal samples grew in both media.

\section{Culling Age Because of Farmer-Suspected Paratuberculosis}

When the age of culling due to the presence of paratuberculosis signs was compared between the vaccinated and nonvaccinated animals, significant differences were observed. As shown in Figure 2A, although no culling due to farmer-suspected paratuberculosis was observed in vaccinated animals $<2.5$ yr old, $13 \%$ of the nonvaccinated animals of the same age were culled due to farmer-suspected paratuberculosis. The peak of the paratuberculosis-associated culling was from 4.5 to $5 \mathrm{yr}$ of age in the vaccinated animals (21\%) and from 3 to $4.5 \mathrm{yr}$ of age $(60 \%)$ in the nonvaccinated animals. Nonvaccinated and vaccinated animals with suspected paratuberculosis were culled at an average age of 3.7 and $4.7 \mathrm{yr}$, respectively. Therefore, vaccination resulted in a 1-yr increase in the productive life of cows sent to slaughter with suspected paratuberculosis compared with nonvaccinated animals culled for the same reason ( $t$-test, $P=0.036)$.

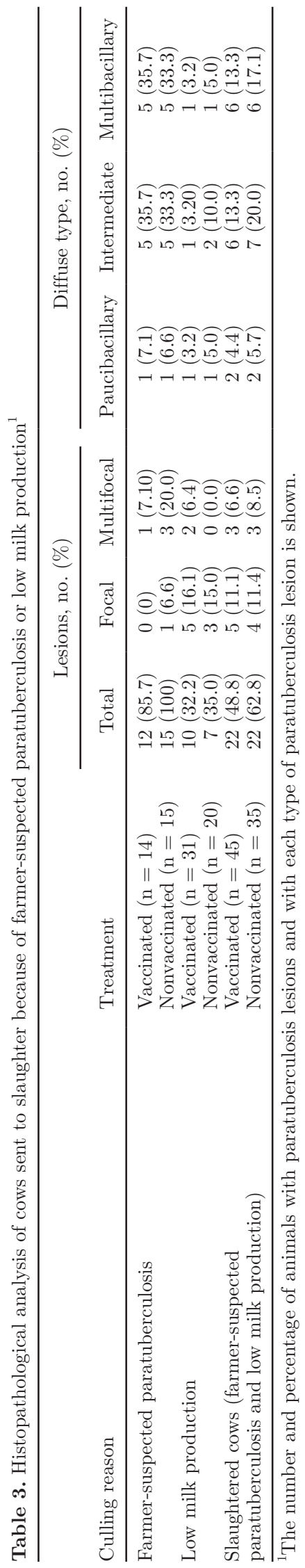

Journal of Dairy Science Vol. 95 No. 2, 2012 
A.

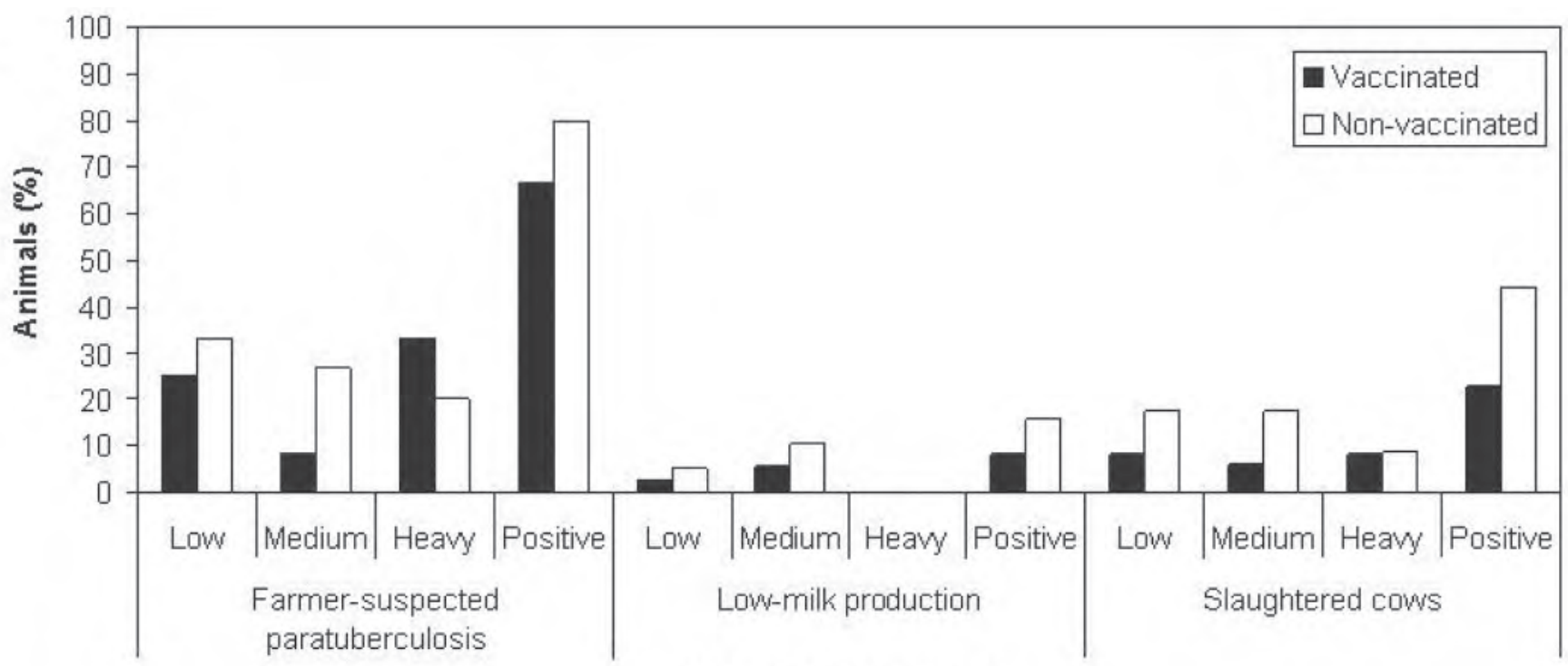

Bacterial load in feces

B.

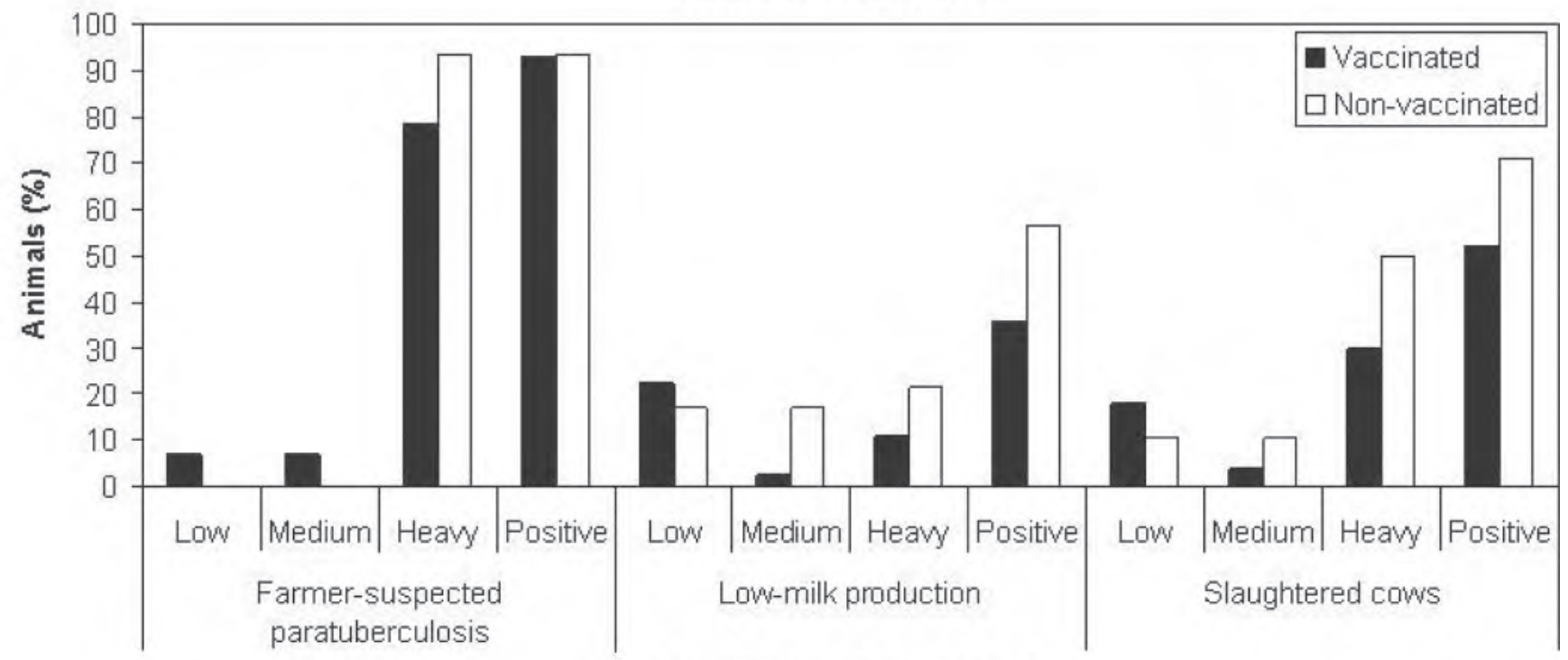

Bacterial load in gut tissues

Figure 1. (A) Bacteriological culture of feces from vaccinated and nonvaccinated cows sent to slaughter because of farmer-suspected paratuberculosis or low milk production. Cows sent to slaughter with farmer-suspected paratuberculosis: vaccinated $(\mathrm{n}=13)$ and nonvaccinated $(\mathrm{n}=$ 15); cows sent to slaughter because of low milk production: vaccinated $(\mathrm{n}=36)$ and nonvaccinated $(\mathrm{n}=19)$. (B) Bacteriological culture of gut tissues from vaccinated and nonvaccinated cows sent to slaughter because of farmer-suspected paratuberculosis or low milk production. Cows sent to slaughter with farmer-suspected paratuberculosis: vaccinated $(\mathrm{n}=14)$ and nonvaccinated $(\mathrm{n}=15)$; cows sent to slaughter because of low milk production: vaccinated $(\mathrm{n}=36)$ and nonvaccinated $(\mathrm{n}=23)$. Bacterial load was classified as low $(<10 \mathrm{cfu})$, medium (10 to $50 \mathrm{cfu})$, and heavy $(>50 \mathrm{cfu})$. The bars show the percentages of vaccinated and nonvaccinated cows with low, medium, heavy bacterial load in feces or tissues.

\section{Overall Losses of All Vaccinated and Nonvaccinated Cows Sent to Slaughter}

We determined the effect of the vaccine on the age at culling of all vaccinated $(\mathrm{n}=332)$ and nonvaccinated animals $(\mathrm{n}=233)$ since the beginning of the trial. For the analysis, nonvaccinated animals were subdivided in 2 categories: animals sent to slaughter with a negative premortem test $(\mathrm{n}=189)$ and animals sent to slaughter with a positive premortem test $(\mathrm{n}=44)$. As shown in Figure 2B, losses occurred more often between 4.5 and $5 \mathrm{yr}$ of age for the vaccinated animals $(17 \%)$, compared with between 4 and $4.5 \mathrm{yr}$ of age for the nonvaccinated animals with a negative premortem test (12\%) and between 3 and 3.5 yr of age for the nonvaccinated animals with a positive premortem test $(30 \%)$. 
A.

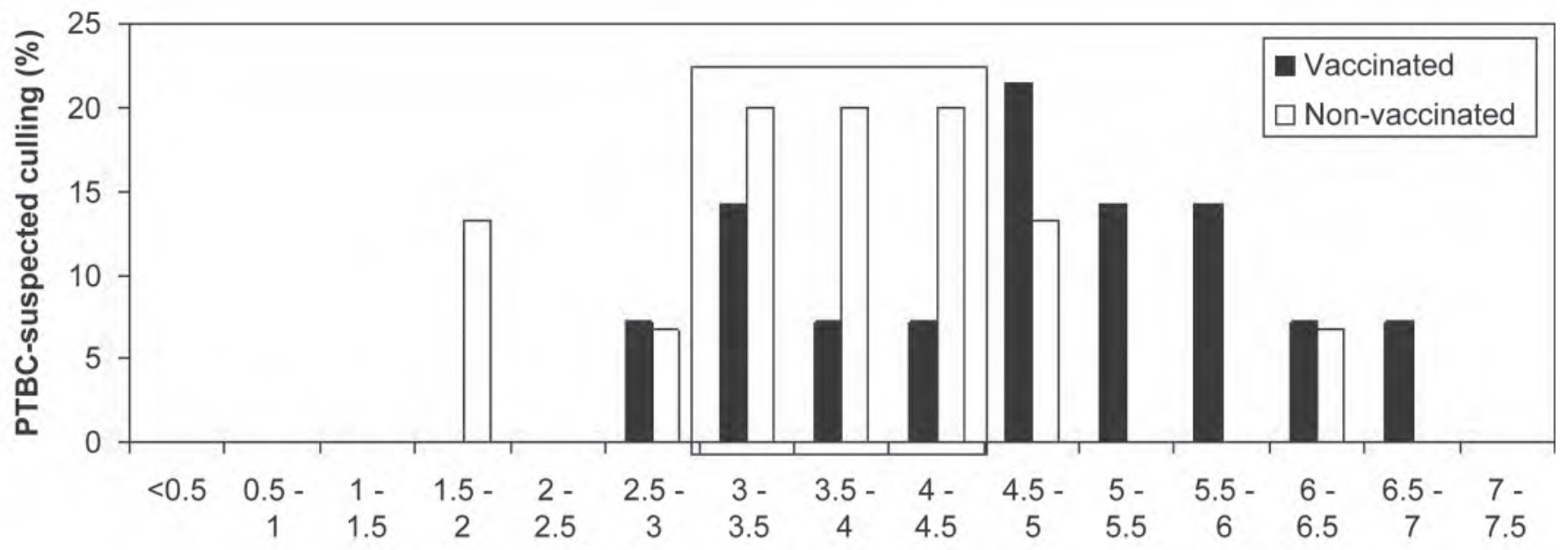

B.

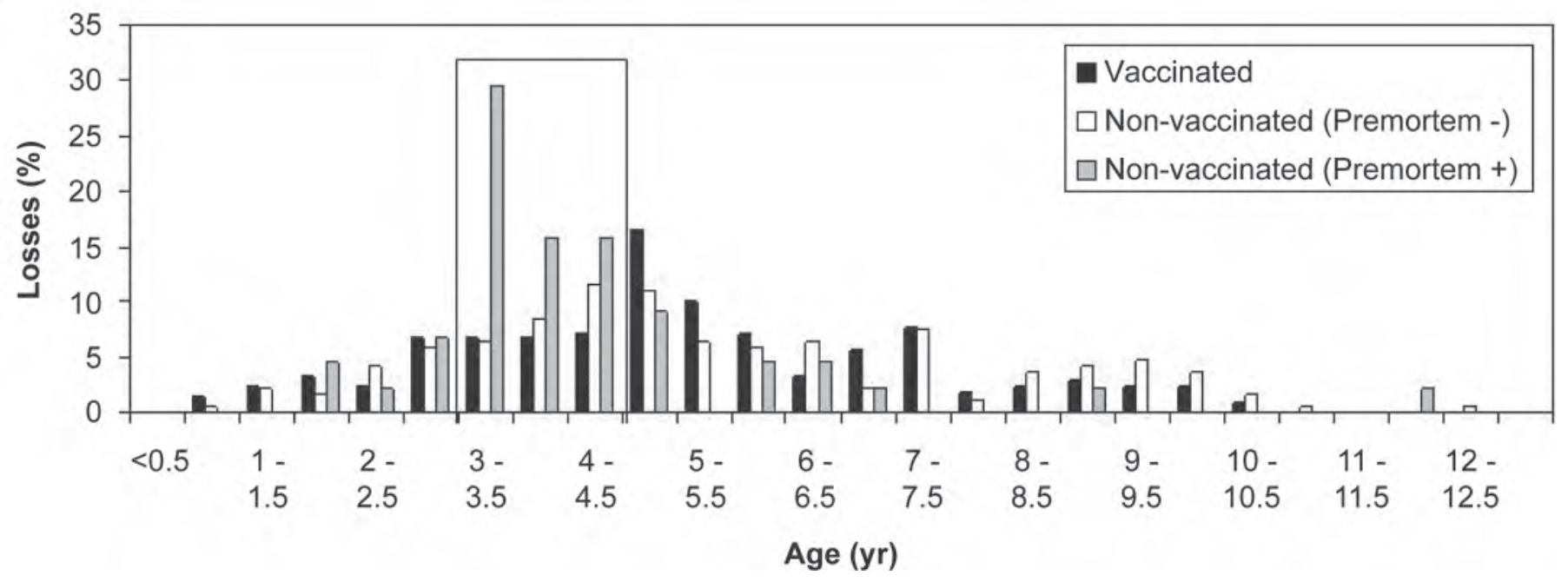

Figure 2. (A) Percentage of vaccinated $(\mathrm{n}=14)$ and nonvaccinated animals $(\mathrm{n}=15)$ that were culled with farmer-suspected paratuberculosis (PTBC-suspected culling) for each age range. (B) Overall losses of all the vaccinated $(\mathrm{n}=332)$ and nonvaccinated animals with a positive $(\mathrm{n}=44)$ or negative premortem test $(\mathrm{n}=189)$ for each age range.

\section{Culling Because of Farmer-Suspected Paratuberculosis at Different Times Postintervention}

We compared the number of vaccinated $(\mathrm{n}=14)$ and nonvaccinated animals $(\mathrm{n}=15)$ sent to slaughter at different times postintervention due to paratuberculosis signs, weight loss, or severe diarrhea (Figure 3). The mean time interval between intervention and culling of the nonvaccinated and vaccinated cows sent to slaughter with paratuberculosis-suspected signs was 12 and 21 mo, respectively. During the first 5 mo postintervention, 6 of the nonvaccinated animals (40\%) were culled due to farmer-suspected paratuberculosis. In contrast, only 1 vaccinated cow (7\%) was sent to slaughter with suspected paratuberculosis during the same period.

\section{Carcass Weight of the Slaughtered Animals with Histopathological Lesions}

The effect of the presence of MAP histopathological lesions on the mean carcass weight of the vaccinated and nonvaccinated animals is shown in Figure 4. Because Jersey cows are smaller than Holsteins the Jersey herd was not included in the analysis. The vaccinated animals had less variability in mean carcass weight (SE: 14.9 vs. 15.4 ) and age at slaughter (SE: 0.36 vs. 0.38 ) than the nonvaccinated animals. Although not statistically significant at this point of the study, a clear trend toward higher weights in vaccinated animals with severe lesions ( $\mathrm{n}=7$; diffuse histopathological forms) was observed compared with the nonvaccinated animals $(\mathrm{n}=16)$. 


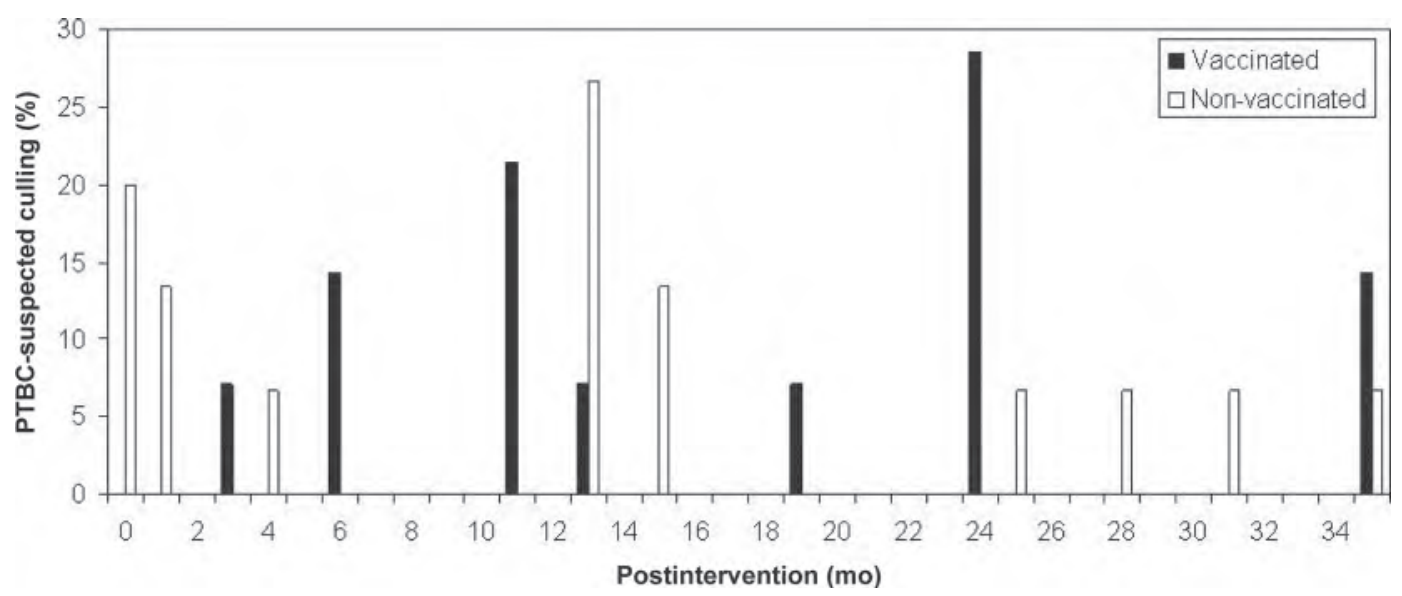

Figure 3. Percentage of vaccinated $(\mathrm{n}=14)$ and nonvaccinated animals $(\mathrm{n}=15)$ that were culled with farmer-suspected paratuberculosis (PTBC-suspected culling) at different times postintervention.

The nonvaccinated animals without histopathological lesions $(\mathrm{n}=14)$ showed higher weights compared with vaccinated cows without lesions $(\mathrm{n}=17$; ANOVA $P=$ 0.011). Mean age at slaughter for the nonvaccinated and vaccinated cows without histopathological lesions was 6.5 and $5.4 \mathrm{yr}$ of age, respectively. The fact that nonvaccinated cows without lesions were kept on the farms for longer than the vaccinated cows might explain the differences in weight at slaughter between both groups. The same is true for the animals with focal lesions. As expected, vaccinated and nonvaccinated animals with focal histopathological lesions or without lesions showed higher weights at slaughter than animals with multifocal or diffuse lesions. In fact, significant differences were observed between the carcass weights of vaccinated $(\mathrm{n}=$ 4 ) or nonvaccinated animals $(n=7)$ with diffuse intermediate lesions versus cows without histopathological lesions (ANOVA $P=0.042$ and $P<0.001$, respectively). Taken together, all cows with diffuse intermediate lesions showed a $33.4 \%(106.7 \mathrm{~kg})$ reduction in their mean weight at slaughter compared with cows without histopathological lesions (ANOVA $P<0.001$ ).

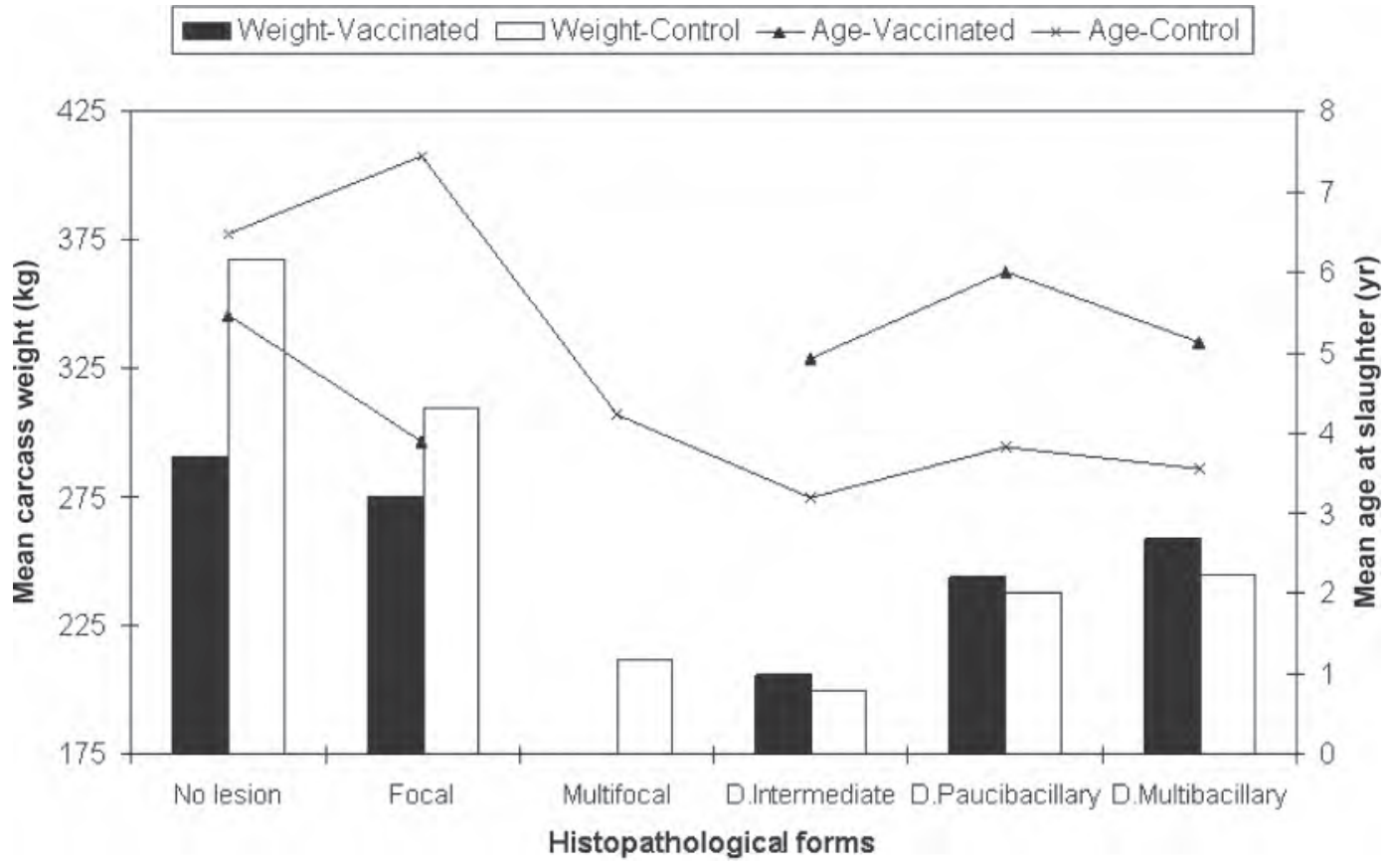

Figure 4. Effect of the presence of histopathological lesions on carcass weight of the vaccinated and nonvaccinated Holstein cows. Open and closed bars show the mean of the weight at slaughter of the vaccinated $(\mathrm{n}=29)$ and nonvaccinated cows $(\mathrm{n}=35)$, respectively. Age at culling for the vaccinated and nonvaccinated animals that showed each type of paratuberculosis-associated lesion in gut tissues is shown with lines. 


\section{DISCUSSION}

In recent years, producers in the Basque Country began reporting significant mortalities from paratuberculosis in cattle and the disease became an industry issue. Economic losses occurred in the infected herds mainly because of premature culling and lower milk production. Producers urgently needed effective and economically attractive control programs to deal with paratuberculosis. Because vaccination had been used with positive results in other countries, producers voluntarily participated in a vaccination program to determine whether a new heat-killed vaccine reduced production losses in their farms. Although similar vaccines have been used for many years in other countries (Larsen et al., 1974, 1978; Larsen and Merkal., 1979; Argenté, 1992; Wentink et al., 1994; Muskens et al., 2002; Emery and Whittington, 2004), a lot of uncertainty still emerges about its effects, particularly whether or not vaccination prevents premature culling of adult cows. In the present study, we evaluated the effects of vaccination of dairy cattle vaccinated as adults, not calves, as has been done in previous vaccination studies.

Thorough evaluation of vaccines against paratuberculosis is time consuming, very expensive, and technically challenging. Taking this last factor into account, several issues regarding the design of our field trial should be considered. First, to gain a more realistic view of the efficacy of the vaccine, vaccinated and nonvaccinated control cows were housed on different farms. Second, we recognized that with only 6 vaccinated herds and 2 control herds it would be difficult to distinguish possible vaccine effects from benefits obtained from the implementation of other management practices designed to reduce the exposure to MAP. In our study, histopathological and microbiological variables were examined in 15 and $16 \%$ of the total culling of the vaccinated and nonvaccinated farms, respectively, since the date of initiation of the trial. However, this limited number of animals appeared to be enough to show statistical differences. This small number of observations emphasizes the importance of extending this field study for several years and updating the evaluations with inclusion of data for the additional years. Third, the dates of initiation of the trial were different on the selected farms. Because our findings were estimated at the cow level, we did not see this as a limitation of our study. To our knowledge, this is the first report to contrast the effects of vaccination on the age of culling and weights at slaughter of adult cows, most of which were likely infected with paratuberculosis before vaccination. Our results are consistent with the hypothesis that immunization with a killed vaccine against MAP significantly reduces both the prevalence of shedders and the frequency of infected cows with MAP in gut tissues at slaughter compared with not vaccinating. We speculate that this reduction in the number of shedders might subsequently reduce the amount of MAP excreted to the environment and thus the spread of the infection to other susceptible herd-mates.

Immunization with the heat-killed vaccine reduced the frequency of the 3.0- to 4.5-yr-old animals being culled due to farmer-suspected paratuberculosis compared with the nonvaccinated animals of the same age. Culling due to farmer-suspected paratuberculosis occurred more frequently between 4.5 and 5 yr of age for the vaccinated animals and between 3 and $4.5 \mathrm{yr}$ of age for the nonvaccinated animals $(60 \%)$. This last age range coincides with the age of slaughter of most of the nonvaccinated animals with a positive premortem test (60\%). Therefore, losses in the 3- to 4.5-yr age range occurred more often when either farmersuspected paratuberculosis or positive-test culling were taken into account. Because of the slow progression of paratuberculosis, animals in this age range are more likely to show signs of paratuberculosis than younger calves (Nielsen and Ersboll, 2006). The mean vaccination age for the 50 vaccinated cows included in our study was 48 mo and the mean time interval between vaccination and slaughter was 15 mo. This implies that most of the cows included in the study reached the potential age of the clinical onset of paratuberculosis at the time of vaccination and were slaughtered many months postvaccination so that the vaccine might exert a positive effect. Therefore, the lower frequency of vaccinated animals with heavy bacterial load in gut tissues suggests a direct therapeutic effect of the vaccine and a significant attenuation of pre-existing infection in adult animals naturally infected with paratuberculosis. These observations of a therapeutic effect of paratuberculosis vaccination are in contrast to the lack of any effect of bacille Calmette-Guérin vaccination on tuberculosis once the pathological process in the lungs is fully engaged (Henao-Tamayo et al., 2009).

Using simulation models, vaccination induced an estimated increase of $1.5 \mathrm{yr}$ at the age at which an animal would become clinically infected and culled (Groenendaal and Galligan, 2003). Our results closely agree with these models because they demonstrated that vaccination extended, by nearly a year, the productive lifespan of vaccinated cattle sent to slaughter with suspected paratuberculosis compared with nonvaccinated animals culled for the same reason. Our analysis revealed a positive effect of the vaccine on carcass weights and on milk production compared with nonvaccinated animals. In fact, a $3.9 \%$ increase in average daily milk production up to $5 \mathrm{yr}$ before and after the beginning of the trial was observed in the vaccinated cows (Juste et 
al., 2009a). Slaughter weights have been reported to be reduced by $10 \%$ in carcasses in which ileocecal lymph nodes, ileum, or rectum were culture-positive for MAP compared with culture-negative cows (Whitlock et al., 1985; Kudahl and Nielsen, 2009). Although not statistically significant at this point of the study, our results suggested that vaccination had a positive effect on carcass weights of the animals with severe histopathological lesions (diffuse histopathological forms) at slaughter compared with the nonvaccinated animals. Interestingly, a significant association was observed between the presence of diffuse intermediate lesions and lower weights at slaughter for both vaccinated and nonvaccinated animals compared with the noninfected cows.

Our study showed a significant reduction in the number of the vaccinated animals with a positive culture of feces or gut tissues. These positive effects were achieved with a single dose of the vaccine given subcutaneously. It has been suggested that eradication of MAP may occur after 4 to 6 yr of vaccination (Argenté, 1992). Therefore, continuation of the vaccination program in the Basque Country for an extended period is needed to have a more precise estimation of the benefits of vaccination against paratuberculosis in terms of reduction of farmer-suspected paratuberculosis and infection prevalence. Vaccination with killed vaccines may not lead to a rapid eradication of paratuberculosis, but it is the most practical and cost-effective approach, with the least intervention, to reduce losses and transmission to animals and human populations. In cattle, the costs of vaccination were estimated at US $\$ 15$ per cow and the benefits at US\$142 per cow (van Schaik et al., 1996). One of the drawbacks of subcutaneous administration of mineral oil-based vaccines is the development of local reactions at the injection site. Our data, however, suggested that the subcutaneous reactions associated with the Silirum MAP vaccine were lesser in cows vaccinated as calves compared with adults and that they disappeared or at least decreased in size in most of the vaccinated animals (data not shown). Hair loss or abscesses at the injection site were not detected.

Although our study was based on 88 slaughtered cows, which represents a small population, our results demonstrated that the vaccine was very effective in the scenario used and provided economic benefits in tuberculosis-free herds. In addition, we recently demonstrated that the vaccine described here did not interfere with the diagnosis of bovine tuberculosis when the comparative intradermal test was used (Garrido et al., 2007).

\section{CONCLUSIONS}

Our results indicate the following significant effects of immunization of adult cattle with a heat-killed vac- cine against paratuberculosis: (1) decreased proportion of fecal shedding cows at slaughter, (2) decreased proportion of cows with positive gut tissue cultures, (3) decreased proportion of cows with higher concentration of MAP in gut tissues, and (4) increased age of culling of cows sent to slaughter with observable clinical signs associated with this disease. Overall, our findings showed that vaccination reduced the premature culling due to paratuberculosis and increased the carcass weights of vaccinated animals compared with that of nonvaccinated cows. Given the period of around $10 \mathrm{yr}$ for the registration of new vaccines, and the low cost, easy delivery, efficacy, and safety of whole-cell vaccines, strategies to control paratuberculosis in the short-term should consider inactivated vaccines such as the one described here, particularly in tuberculosis-free areas. In addition to vaccination, management improvements (e.g., separating calves from adult cattle, increasing farm hygiene) are also recommended.

\section{ACKNOWLEDGMENTS}

This study was funded by grants from the Ministerio de Ciencia e Innovación (Spain) (AGL2006-14315CO2) and from the Instituto Nacional de Investigación y Tecnología Agraria y Alimentaria (Spain) (RTA200500147-00-00). Salaries for Marta Alonso-Hearn and Joseba Garrido were partly covered by the INIA program for the incorporation of $\mathrm{PhD}$ graduates to research institutions. Patricia Vázquez has a Formación de Personal Investigador fellowship from the Ministerio de Ciencia e Innovación. We are grateful to Carlos Boix and Jose M. Plazaola from the Animal Health Service at the Diputacion Foral de Gipuzkoa for supporting the vaccination trial. We are also grateful to Iñaki Arrazola and Blanca Ocabo from the Animal Health Service at the Diputacion Foral de Bizkaia for their support in the follow-up of the nonvaccinated herds. The authors thank the cattle owners, and the veterinary practitioners who helped with the immunizations and collection of samples (Isidro Aristi, Xabier Kaminos, Juan R. Urteaga, Francisco J. Mendizabal, and Apolinar Insausti). We express our thanks to the veterinarians and staff at Donosti, Zestona, and Bilbao slaughterhouses for their help in collecting postmortem samples. Cristina Espiga and Juan Antonio Obispo (IKT, S.A.) are thanked for providing access to the carcass weights data. We thank the staff at the Department of Histopathology (NEIKER-Tecnalia) for excellent technical assistance. We are grateful to Kyle P. Hearn for careful editing of the manuscript.

\section{REFERENCES}

Argenté, G. 1992. Efficiency of vaccination and other control measures estimated by fecal culturing in a regional program. Pages 495-503 
in Proc. III International Colloquium on Paratuberculosis. R. J. Chiodini, and J. M. Kreeger, ed. International Association for Paratuberculosis Inc., Providence, RI.

Chamberlin, W., D. Y. Graham, K. Hulten, H. M. El-Zimaity, M. R. Schwartz, S. Naser, I. Shafran, and F. A. El-Zaatari. 2001. Review article: Mycobacterium avium ssp. paratuberculosis as one cause of Crohn's disease. Aliment. Pharmacol. Ther. 15:337-346.

Chiodini, R. J., H. J. Van Kruiningen, and R. S. Merkal. 1984. Ruminant paratuberculosis (Johne's disease): The current status and future prospects. Cornell Vet. 74:218-262.

Collins, M. T. 2003. Paratuberculosis: Review of present knowledge. Acta Vet. Scand. 44:217-221.

Collins, M. T., S. J. Wells, K. R. Petrini, J. E. Collins, R. D. Schultz, and R. H. Whitlock. 2005. Evaluation of five antibody detection tests for diagnosis of bovine paratuberculosis. Clin. Diagn. Lab. Immunol. 12:685-692.

Daniels, M. J., M. R. Hutchings, and A. Greig. 2003. The risk of disease transmission to livestock posed by contamination of farm stored feed by wildlife excreta. Epidemiol. Infect. 130:561-568.

Emery, D. L., and R. J. Whittington. 2004. An evaluation of mycophage therapy, chemotherapy and vaccination for control of $M y-$ cobacterium avium ssp. paratuberculosis infection. Vet. Microbiol. 104:143-155.

Garrido, J. M., E. Molina, M. V. Geijo, J. M. Plazaola, I. Sevilla, and R. A. Juste. 2007. Preliminary evaluation of a field trial on the use of vaccination in dairy cattle farms with paratuberculosis. Pages 204-207 in Proc. IX Int. Colloq. Paratuberculosis. S. S. Nielsen, ed. International Association for Paratuberculosis Inc., Derio, Spain.

González, J., M. V. Geijo, C. García-Pariente, A. Verna, J. M. Corpa, L. E. Reyes, M. C. Ferreras, R. A. Juste, J. F. Garcia-Marin, and V. Pérez. 2005. Histopathological classification of lesions associated with natural paratuberculosis infection in cattle. J. Comp. Pathol. 133:184-196.

Groenendaal, H., and D. T. Galligan. 2003. Economic consequences of control programs for paratuberculosis in midsize dairy farms in the United States. J. Am. Vet. Med. Assoc. 223:1757-1763.

Henao-Tamayo, M., G. S. Palaniswamy, E. E. Smith, C. A. Shanley, B. Wang, I. M. Orme, R. J. Basaraba, N. M. DuTeau, and D. Ordway. 2009. Post-exposure vaccination against Mycobacterium tuberculosis. Tuberculosis (Edinb.) 89:142-148.

Juste, R. A., M. Alonso-Hearn, E. Molina, M. Geijo, P. Vazquez, I. Sevilla, and J. M. Garrido. 2009a. Significant reduction in bacterial shedding and improvement in milk production in four dairy farms after the use of a new inactivated paratuberculosis vaccine. BMC Res. Notes 2:233.

Juste, R. A., N. Elguezabal, J. M. Garrido, A. Pavon, M. V. Geijo, I. Sevilla, J. L. Cabriada, A. Tejada, F. Garcia-Campos, R. Casado, I. Ochotorena, A. Izeta, and R. J. Greenstein. 2008. On the prevalence of $M$. avium subspecies paratuberculosis DNA in the blood of healthy individuals and patients with inflammatory bowel disease. PLoS ONE 3:e2537.

Juste, R. A., N. Elguezabal, A. Pavon, J. M. Garrido, M. Geijo, I. Sevilla, J. L. Cabriada, A. Tejada, F. Garcia-Campos, R. Casado, I. Ochotorena, and A. Izeta. 2009b. Association between Mycobacterium avium ssp. paratuberculosis DNA in blood and cellular and humoral immune response in inflammatory bowel disease patients and controls. Int. J. Infect. Dis. 13:247-254.

Juste, R. A., J. F. García Marín, B. Peris, C. Sáez de Ocáriz, and J. J. Badiola. 1994. Experimental infection of vaccinated and nonvaccinated lambs with Mycobacterium paratuberculosis. J. Comp. Pathol. 110:185-194.

Juste, R. A., J. C. Marco, C. Saez de Ocariz, and J. J. Aduriz. 1991. Comparison of different media for the isolation of small ruminant strains of Mycobacterium paratuberculosis. Vet. Microbiol. 28:385-390.

Köhler, H., H. Gyra, K. Zimmer, K. G. Drager, B. Burkert, B. Lemser, D. Hausleithner, K. Cubler, W. Klawonn, and R. G. Hess. 2001.
Immune reactions in cattle after immunization with a Mycobacterium paratuberculosis vaccine and implications for the diagnosis of M. paratuberculosis and M. bovis infections. J. Vet. Med. B Infect. Dis. Vet. Public Health 48:185-195.

Körmendy, B. 1994. The effect of vaccination on the prevalence of paratuberculosis in large dairy herds. Vet. Microbiol. 41:117-125.

Kudahl, A. B., and S. S. Nielsen. 2009. Effect of paratuberculosis on slaughter weight and slaughter value of dairy cows. J. Dairy Sci. 92:4340-4346.

Larsen, A. B., and R. S. Merkal. 1979. Effectiveness of killed virulent cell vaccine against paratuberculosis. Proc. Annu. Meet. U. S. Anim. Health Assoc. 83:480-484.

Larsen, A. B., R. S. Merkal, and H. W. Moon. 1974. Evaluation of a paratuberculosis vaccine given to calves before infection. Am. J. Vet. Res. 35:367-369.

Larsen, A. B., A. I. Moyle, and E. M. Himes. 1978. Experimental vaccination of cattle against paratuberculosis (Johne's disease) with killed bacterial vaccines: A controlled field study. Am. J. Vet. Res. 39:65-69.

Manning, E. J., and M. T. Collins. 2001. Mycobacterium avium ssp. paratuberculosis: Pathogen, pathogenesis and diagnosis. Rev. Sci. Tech. 20:133-150.

Moss, M. T., J. D. Sanderson, M. L. V. Tizard, J. Hermon-Taylor, F. El-Zaatari, D. C. Markesich, and D. Y. Graham. 1992. Polymerase chain reaction detection of Mycobacterium paratuberculosis and Mycobacterium avium subsp silvaticum in long term cultures from Crohn's disease and control tissues. Gut 33:1209-1213.

Muskens, J., F. van Zijderveld, A. Eger, and D. Bakker. 2002. Evaluation of the long-term immune response in cattle after vaccination against paratuberculosis in two Dutch dairy herds. Vet. Microbiol. 86:269-278.

Nielsen, S. S., H. Bjerre, and N. Toft. 2008. Colostrum and milk as risk factors for infection with Mycobacterium avium subspecies paratuberculosis in dairy cattle. J. Dairy Sci. 91:4610-4615.

Nielsen, S. S., and A. K. Ersboll. 2006. Age at occurrence of Mycobacterium avium subspecies paratuberculosis in naturally infected dairy cows. J. Dairy Sci. 89:4557-4566.

Ott, S. L., S. J. Wells, and B. A. Wagner. 1999. Herd-level economic losses associated with Johne's disease on US dairy operations. Prev. Vet. Med. 40:179-192.

Pierce, E. S. 2009. Possible transmission of Mycobacterium avium subspecies paratuberculosis through potable water: Lessons from an urban cluster of Crohn's disease. Gut Pathog. 1:17.

Rosenfeld, G., and B. Bressler. 2010. Mycobacterium avium paratuberculosis and the etiology of Crohn's disease: A review of the controversy from the clinician's perspective. Can. J. Gastroenterol. 24:619-624. (Review).

Sherman, D. M., J. M. Gay, D. S. Bouley, and G. H. Nelson. 1990. Comparison of the complement-fixation and agar gel immunodiffusion tests for diagnosis of subclinical bovine paratuberculosis. Am. J. Vet. Res. 51:461-465

Smith, B. P., ed. 1990. Large Animal Internal Medicine. The C. V. Mosby Company, St. Louis, MO.

Sweeney, R. W.. R. H. Whitlock, and E. A. Rosenberger. 1996. Transmission of paratuberculosis. Vet. Clin. North Am. Food Anim. Pract. 12:305-312. (Review).

van Schaik, G., C. H. J. Kalis, G. Benedictus, A. A. Dijkhuizen, and R. B. M. Huirne. 1996. Cost-benefit analysis of vaccination against paratuberculosis in dairy cattle. Vet. Rec. 139:624-627.

Wentink, G. H., J. H. Bongers, A. A. P. A. Zeeuwen, and F. H. J. Jaartsveld. 1994. Incidence of paratuberculosis after vaccination against M. paratuberculosis in two infected dairy herds. Zentralbl. Veterinarmed. B 41:517-522.

Whitlock, R. H., L. T. Hutchingson, R. Merkal, L. T. Glickman, C. Rossiter, S. Harmon, P. Spencer, J. Fetrow, J. Bruce, C. E. Benson, and J. Dick. 1985. Prevalence and economic consideration of Johne's disease in the northeastern U.S. Proc. US Anim. Health Assoc. 89:484-488. 